Pathologe $2019 \cdot 40: 36-45$

https://doi.org/10.1007/s00292-019-0567-6

Online publiziert: 29. Januar 2019

(c) Der/die Autor(en) 2019

\section{Redaktion}

H. A. Baba, Essen

CrossMark

\section{S. F. Lax}

Institut für Klinische Pathologie und Molekularpathologie, LKH Graz II, Standort West, Akademisches Lehrkrankenhaus der Medizinischen Universität Graz, Graz, Österreich

\title{
Mesenchymale und gemischte Uterustumoren
}

\section{Aktuelle Übersicht und praktische Aspekte}

Im Bereich des Uterus können grundsätzlich alle mesenchymalen Tumoren vorkommen (『Tab. 1). Im Gegensatz zu den glattmuskulären Tumoren sind andere mesenchymale Tumoren aber sehr selten. Als Faustregel gilt, dass mehr als $90 \%$ aller mesenchymalen Tumoren gutartig sind bzw. eine glattmuskuläre (leiomyogene) Differenzierung aufweisen. Die zweithäufigste Gruppe der mesenchymalen Uterustumoren, Tumoren des Endometriumstromas, sind ebenso wie alle anderen mesenchymalen Tumortypen und gemischte Tumoren des Uterus sehr selten [1]. Für alle Uterussarkome gibt es eine mittlerweile aktualisierte TNM-Klassifikation [2].

\section{Mesenchymale Tumoren}

$\mathrm{Zu}$ den mesenchymalen Tumoren des Uterus zählen vor allem glattmuskuläre (leiomyogene) Tumoren und Tumoren, die sich vom Endometriumstroma herleiten, aber auch eine Reihe anderer seltener Neoplasien ( $\bullet$ Tab. 1; $[3,4]$ ).

\section{Glattmuskuläre Tumoren}

Leiomyome sind die häufigsten Tumoren des Uterus und betreffen jede vierte bis fünfte Frau, typischerweise im vierten und fünften Lebensjahrzehnt. Die typische Lokalisation ist das Corpus uteri. Ein Befall der Zervix ist dem gegenüber wesentlich seltener [1]. Leiomyome finden sich meist innerhalb des Myometriums (intramural), seltener submukös bzw. subserös, wobei die beiden letzteren Lokalisationen häufig mit Durchblutungsstörungen und Nekrosen asso- ziiert sein können. Während subseröse Leiomyome selten Probleme in Form einer akuten abdominellen Schmerzsymptomatik verursachen, führen submuköse Leiomyome häufig zu vaginalen Blutungen [3].

\section{Diagnostische Probleme von glattmuskulären Uterustumoren}

Die meisten leiomyogenen Tumoren sind diagnostisch problemlos und lassen sich als gutartige Leiomyome diagnostizieren [3]. Hochgradig polymorphzellige Tumoren sind wiederum eindeutig als Sarkome zu erkennen. Es gibt aber immer wieder diagnostisch anspruchsvolle Fälle, die hinsichtlich ihrer Dignität nicht eindeutig eingeordnet werden können. Für diese wurde eine Zwischengruppe mit unsicherem biologischem Verhalten eingeführt, die als glattmuskuläre Tumoren unsicherer maligner Potenz (engl. „smooth muscle tumors of uncertain malignant potential/STUMP") oder atypische glattmuskuläre Neoplasie (engl. „atypical smooth muscle neoplasm") bezeichnet wird [3]. Für die problematischen Fälle gibt es leider eingeschränkte Evidenz, da ihre Anzahl selbst in größeren Studien klein ist und sie oft noch in Untergruppen aufgeteilt wurden [5]. Zur Komplexität der Diagnostik glattmuskulärer Uterustumoren hat auch der Wandel der Malignitätskriterien im Laufe der Zeit beigetragen [1]. Historisch gesehen spielte insbesondere in Europa die Anzahl der Mitosen für die Dignität von glattmuskulären Tumoren eine große Rolle. In den USA hatte hingegen die zelluläre Atypie eine größere Bedeutung (,No atypia, no sar- coma“). Ein Meilenstein war vor etwa 25 Jahren die Publikation der StanfordGruppe, die neben zellulären Atypien und Mitosen auch der Tumorzellnekrose eine wesentliche Bedeutung zukommen ließ [5]. Die Trias Atypien, Tumorzellnekrosen und Mitosen fand Eingang in die WHO-Klassifikation 2003 [6]. In der Folge wurden aber die Probleme der Reproduzierbarkeit der Tumorzellnekrose deutlich. Dem hat die WHO-Klassifikation 2014 auch Rechnung getragen und speziell der zellulären Atypie eine größere Bedeutung beigemessen [3]. Für die Diagnose eines Leiomyosarkoms sind sowohl zelluläre Atypie als auch mitotische Aktivität erforderlich (• Abb. 1). Dabei ist ein mäßiger bis hoher Atypiegrad erforderlich. Die für eine Sarkomdiagnose erforderliche mitotische Aktivität wurde nicht näher definiert, wird aber in der Regel als $\geq 10$ Mitosen pro $10 \mathrm{HPF}$ angesehen. In Abschnitten mit sehr ausgeprägter Atypie können Mitosen fehlen oder schwierig aufzufinden sein. Auch die Tumorzellnekrosen, die sich in etwa einem Drittel der Leiomyosarkome finden und durch einen abrupten Übergang von vitalem zu nichtvitalem Tumorgewebe charakterisiert sind, zählen zu den Malignitätskriterien. Die Tumorzellen sind dabei oft perivaskulär angeordnet. Innerhalb der Tumorzellnekrose finden sich Schatten der Tumorzellen mit auffallend hyperchromatischen Zellkernen. In Endometriumbiopsien bzw. Abradaten finden sich nicht selten Nekrosen, die zwar ischämischer Natur sind, aber durch Kernschatten und sog. geografische Struktur Tumorzellnekrosen stark ähneln können. Im Randbereich der Ne- 
Tab. 1 Klassifikation der mesenchymalen und gemischten Tumoren des Uterus (WHO 2014)

Tumortyp

ICD-0-Code

\begin{tabular}{l|l}
\hline Glattmuskuläre Tumoren & \\
\hline Leiomyom und Varianten & $8890 / 0$ \\
\hline Zellreiches Leiomyom & $8892 / 0$ \\
\hline Leiomyom mit bizarren Zellkernen & $8893 / 0$ \\
\hline Mitotisch aktives Leiomyom & $8890 / 0$ \\
\hline Hydropisches Leiomyom & $8890 / 0$ \\
\hline Apoplektisches Leiomyom & $8890 / 0$ \\
\hline Lipomatöses Leiomyom (Lipoleiomyom) & $8891 / 0$ \\
\hline Epitheloides Leiomyom & $8896 / 0$ \\
\hline Myxoides Leiomyom & $8890 / 0$ \\
\hline Dissezierendes (kotyledonoides) Leiomyom & $8890 / 1$ \\
\hline Diffuse Leiomyomatose & $8890 / 1$ \\
\hline Intravenöse Leiomyomatose & $8898 / 1$ \\
\hline Metastasierendes Leiomyom &
\end{tabular}

Glattmuskuläre Tumoren unsicheren malignen Potenzials (STUMP)/atypische glattmuskuläre Neoplasie

Leiomyosarkom

$8890 / 3$

Epitheloides Leiomyosarkom

$8891 / 3$

Myxoides Leiomyosarkom

$8896 / 3$

Tumoren des Endometriumstromas und verwandte Tumoren

Gut differenziertes (low-grade)-Sarkom des Endometriumstromas (ESS)

$8831 / 0$

Schlecht differenziertes (high-grade) Sarkom des Endometriumstromas (ESS)

$8930 / 0$

Undifferenziertes Sarkom des Uterus

$8805 / 3$

Keimstrangartiger Tumor des Uterus (UTROSCT)

$8590 / 1$

Unterschiedliche mesenchymale Tumoren

Rhabdomyosarkom

$8900 / 3$

Perivaskulärer Epitheloidzelltumor, benigne

$8714 / 0$

Perivaskulärer Epitheloidzelltumor, maligne

$8714 / 3$

Andere mesenchymale Tumoren

Gemischte epitheliale und mesenchymale Tumoren

Adenomyom

$8932 / 0$

Atypisches polypoides Adenomyom (APAM)

$8932 / 0$

Adenofibrom

$9013 / 0$

Adenosarkom

$8933 / 3$

Karzinosarkom

$8980 / 3$

krosen befindliche Mitosen und zelluläre Atypien können diagnostische Probleme verstärken. Weitere diagnostische Kriterien für Malignität sind Gefäßinvasion, die in bis zu 10-20\% der Fälle vorkommt, und infiltratives Wachstum ins umgebende Myometrium. Ein Gradingsystem existiert nicht, da alle Leiomyosarkome per se als schlecht differenziert („highgrade") gelten.

Der Großteil der Leiomyosarkome weist typischerweise eine auch als konventionell bezeichnete spindelige Histologie auf. Viel seltener sind eine
CAP-Jahrestagung vorliegt [7]. Obwohl in Leiomyosarkomen eine Reihe genomischer Veränderungen, insbesondere komplexe numerische und strukturelle chromosomale Aberrationen gefunden wurden, gibt es bis dato weder pathognomonische und diagnostisch signifikante noch therapeutisch relevante genomische Alterationen. Eine MED12Amplifikation findet sich sowohl in Leiomyomen als auch Leiomyosarkomen. Mittels der Array-CGH („array-based comparative genomic hybridization“) konnte eine Unterscheidung benigner von malignen glattmuskulären Tumoren getroffen und damit die STUMP-Kategorie vermieden werden [8]. Es scheint, dass mittels Genexpressionsprofilen Leiomyosarkome von Stromasarkomen des Endometriums unterschieden werden können [9].

Die Prognose von Leiomyosarkomen ist ungünstig, insbesondere in den Stadien III und IV. Das Tumorstadium ist der stärkste prognostische Parameter [2].

\section{Leiomyomvarianten}

Einige der zahlreichen Leiomyomvarianten verdienen eine genauere Betrachtung [10]. Zellreiche Leiomyome sind durch eine deutlich höhere Zelldichte als das umgebende Myometrium charakterisiert und können eine rundzellige Morphologie sowie zahlreiche Mitosen (mitosereiche Leiomyome) aufweisen. Sie müssen von Stromaknoten des Endometrium abgegrenzt werden. Dazu sind sowohl Immunhistochemie (Positivität für Muskelmarker wie Desmin und Caldesmon) als auch Histomorphologie (Fehlen der kleinen arteriolenartigen Blutgefäße) hilfreich. Leiomyome mit bizarren Zellkernen sind durch entweder herdförmig oder diffus angeordnete, deutlich polymorphe Zellkerne charakterisiert, wobei sich im Hintergrund meist ein unauffälliges glattmuskuläres Muster findet (• Abb. 2). Die bizarren Zellkerne sind hyperchromatisch, ihr Chromatin ist oft verwaschen. Der Mitosegehalt ist gering, Karyorrhexisfiguren können atypische Mitosefiguren imitieren. Die Ki67-Immunhistochemie kann einen Anhaltspunkt bieten, exakte Richtwerte gibt es aber nicht. Bei Vorhandensein von Nekrosen kann die Abgrenzung zu Leio- 
myosarkomen Schwierigkeiten bereiten, zumal diese Nekrosen zwar einen infarktartigen ischämischen Aspekt zeigen, durch Kernschatten aber eine Ähnlichkeit mit Tumorzellnekrosen bestehen kann. Eine vor kurzem veröffentlichte Studie an beinahe 60 Fällen hat einen durchweg gutartigen Verlauf gezeigt [11]. Mitosereiche Leiomyome sind typisch für die fertile Lebensphase und können mit einer Hormontherapie vergesellschaftet sein. Sie weisen häufig einen Mitosegehalt von >10/10 HPF auf. Eine besondere Variante stellen Leiomyome mit einem Defizit an Fumarase bzw. Fumarat-Hydratase (FH) dar [12]. Diese Knoten, die sich meist bei jungen Frauen finden, weisen oft eine deutliche Kernpolymorphie und einen erhöhten Zellreichtum auf, wobei differenzialdiagnostisch Leiomyome mit bizarren Zellkernen im Raum stehen (- Abb. 3). Der Mangel an FumaratHydratase kann immunhistochemisch nachgewiesen werden. Histomorphologisch auffallend sind eine palisadenartige Anordnung der Zellkerne und hirschgeweihartige Blutgefäße. FH-defiziente Leiomyome finden sich in etwa $0,4-1,6 \%$ aller nichtselektierten Leiomyome ohne Atypien sowie in 1,8\% aller zellreichen Leiomyome und etwa einem Drittel aller Leiomyome mit bizarren Zellkernen [13]. Eine FH-Defizienz konnte bis dato hingegen noch nicht in Leiomyosarkomen nachgewiesen werden [12].

Auch GnRH-Agonisten, wie sie zur Behandlung der Endometriose, des Mammakarzinoms, aber auch von Myomen sowie zur In-vitro-Fertilisation eingesetzt werden, können zu einem verstärkten Zellreichtum glattmuskulärer Tumoren führen, verbunden mit dicht liegenden und überlappenden Zellkernen [14]. Tranexamsäure, die gegen Menorrhagien eingesetzt wird, führt wiederum zu infarktartigen Nekrosen, die nicht mit Tumorzellnekrosen verwechselt werden dürfen [15]. Im Zuge einer Gestagenmedikation kann es ebenfalls zu infarktartigen Nekrosen, erhöhtem Zellreichtum, mitotischer Aktivität und Kernauffälligkeiten in leiomyogenen Tumoren kommen, bis hin zur Schwierigkeit der Abgrenzung zu STUMP und Leiomyosarkom [16].

Pathologe 2019 · 40:36-45 https://doi.org/10.1007/s00292-019-0567-6

(c) Der/die Autor(en) 2019

S. F. Lax

\section{Mesenchymale und gemischte Uterustumoren. Aktuelle Übersicht und praktische Aspekte}

\section{Zusammenfassung}

Gutartige Leiomyome sind die häufigsten mesenchymalen Uterustumoren, während Sarkome des Uterus sehr selten sind. Die häufigsten Sarkome sind Leiomyosarkome gefolgt von Sarkomen des Endometriumstromas. Leiomyosarkome sind durch eine deutliche Kernpolymorphie und einen hohen Mitosegehalt charakterisiert, daneben können sie Tumorzellnekrosen, ein invasives Wachstum sowie Gefäßeinbrüche aufweisen. In Fällen einer diagnostischen Unsicherheit sollte nur in Ausnahmefällen die Kategorie eines glattmuskulären Tumors unsicherer maligner Potenz (STUMP) zur Anwendung gelangen. Neben den Low-grade-Sarkomen und den Knoten des Endometriumstromas wurde 2014 von der WHO eine High-gradeSarkomkategorie (ESS) wieder etabliert. Highgrade ESS zeigen eine typische fibromyxoide oder rundzellige Histomorphologie, ein myoinvasives Wachstum, Immunreaktivität für Cyclin D1 und BCOR und charakteristische Fusionen im Bereich der YWHAE- bzw. BCORGene. Die sehr seltenen undifferenzierten
Sarkome des Uterus müssen aufgrund ihrer Überlappung mit High-grade-ESS neu definiert werden. Keimstrangartige Tumoren des Uterus (UTROSCT) sind selten maligne, müssen aber von Endometriumkarzinomen abgegrenzt werden. Gemischte epitheliale und mesenchymale Uterustumoren sind selten, Karzinosarkome dabei häufiger als Adenosarkome. Beim Adenosarkom ist das Erkennen einer hochmalignen Sarkomkomponente prognostisch wichtig. Karzinosarkome sind histomorphologisch heterogen, aber klonal und werden biologisch wie undifferenzierte Karzinome eingestuft. Molekulare Diagnostik wird in Zukunft vermehrt die Klassifikation seltener und ungewöhnlicher mesenchymaler Uterustumoren unterstützen.

\section{Schlüsselwörter}

Uterussarkome - Gemischte Uterustumoren - Leiomyome · Stromasarkome des Endometriums $\cdot$ Karzinosarkome

\section{Mesenchymal and mixed uterine tumors. Current overview and practical aspects}

\section{Abstract}

Benign leiomyomas are the most frequent mesenchymal tumors of the uterus. In contrast, uterine sarcomas are very rare. Leiomyosarcomas are the most frequent sarcomas followed by endometrial stromal sarcomas (ESS). Leiomyosarcomas are characterized by marked nuclear atypia and high mitotic count and may also show tumor cell necrosis and myometrial and vascular invasion. For cases of diagnostic uncertainty, the category of smooth muscle tumor of uncertain malignant potential (STUMP) may be considered but should be rarely used. Besides low-grade ESS and stromal nodules, a category of high-grade ESS was reconsidered by the WHO in 2014. High-grade ESS are characterized by fibromyxoid and round cell histology, myoinvasive growth, and immunoreactivity for cyclin D1 and $B C O R$ and distinct gene fusions involving $Y W H A E$ and $B C O R$, respectively. The very rare undifferentiated uterine sarcomas need to be redefined due to overlap with highgrade ESS. Uterine tumors resembling ovarian sex cord tumors (UTROSCT) rarely behave malignant, but need to be distinguished from endometrial carcinomas. Mixed epithelial and mesenchymal tumors of the uterus are rare with carcinosarcomas occurring more frequently than adenosarcomas. For prognosis of adenosarcomas the recognition of sarcomatous overgrowth is crucial. Carcinosarcomas are histologically heterogeneous although genetically clonal; biologically they are considered as undifferentiated carcinomas. There will be an increasing importance of molecular pathology for the classification of rare and unusual mesenchymal uterine tumors.

\section{Keywords}

Uterine sarcoma - Mixed uterine tumors . Leiomyoma - Endometrial stromal sarcoma . Carcinosarcoma 

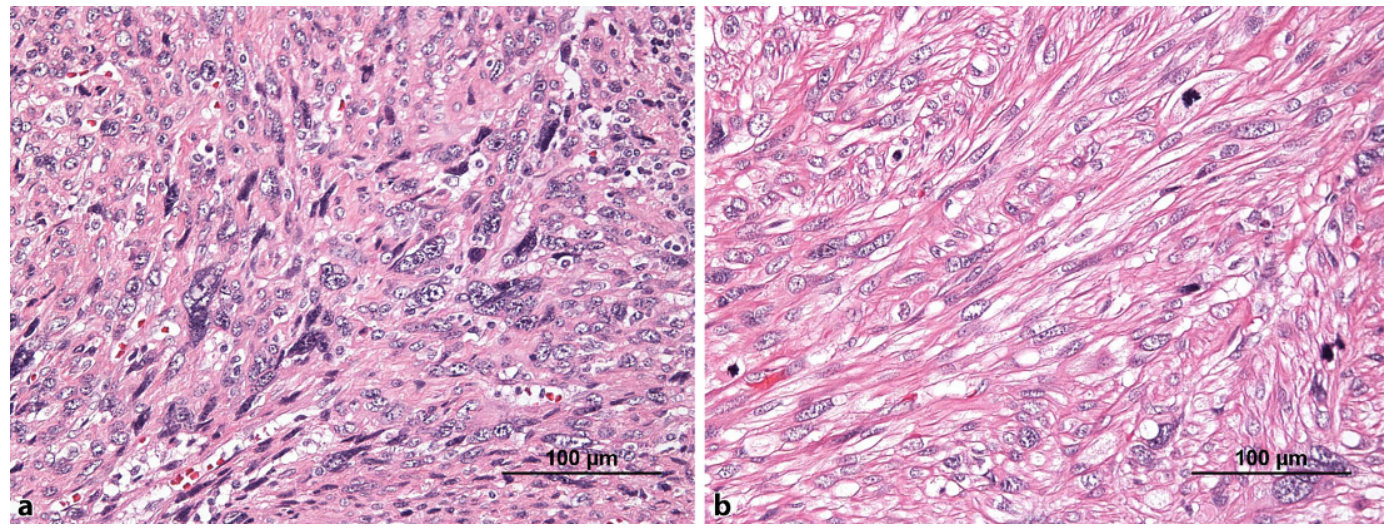

Abb. $1<$ Leiomyosarkom. Hochgradige diffuse Atypie und Polymorphie (a) sowie reichlich Mitosen (b). HEFärbung
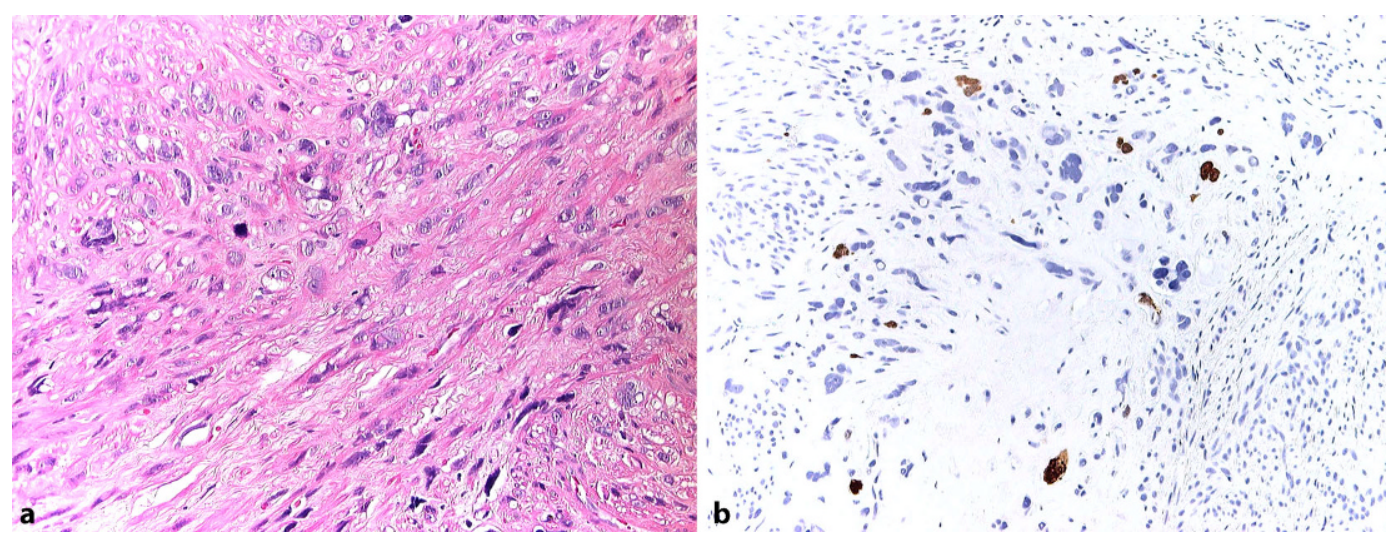

Abb. $2 \triangleleft$ Leiomyom mit bizarren Zellkernen („bizarres Leiomyom"). a Ein Teil der Zellen ist deutlich atypisch bei reichlich unauffälligen Zellen im Hintergrund. HE-Färbung. b Niedriger Ki67-Färbeindex bei einem Teil der bizarren Zellkerne. DAB-Färbung
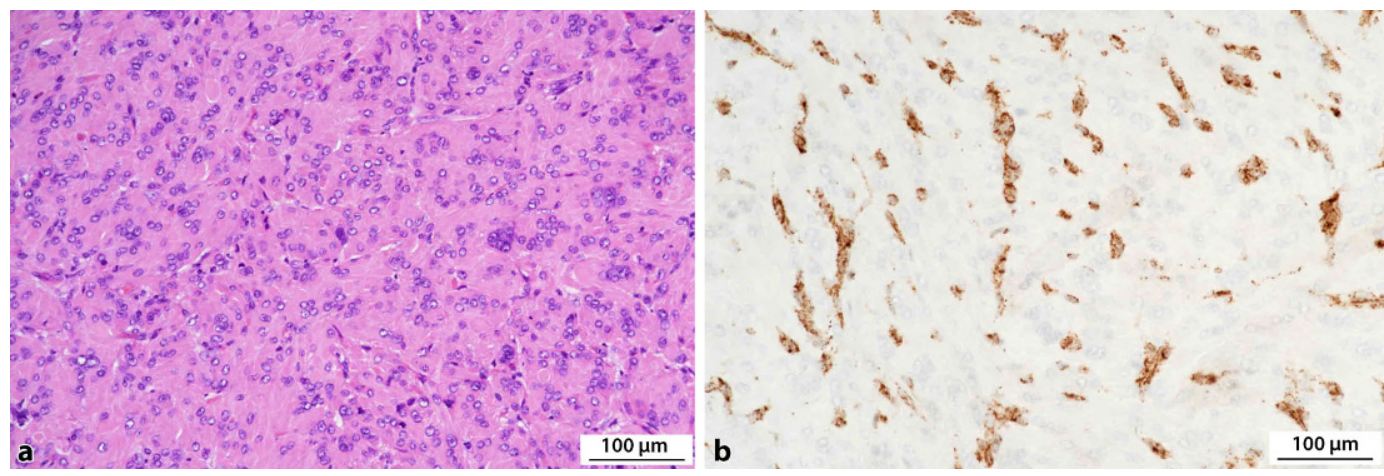

Abb. $3<$ Leiomyom mit Fehlen der Fumarat-Hydratase/Fumarase (FH). a Mäßiggradige Kerngrößenschwankungen und Ausbildung mehrkerniger Zellen. HE-Färbung. b Die Immunreaktivität für FH fehlt im Bereich der glatten Muskelzellen, ist aber im Bereich der typischen verzweigten Gefäße erhalten. DAB-Färbung

\section{Glattmuskuläre Tumoren unsicheren malignen Potenzials (STUMP)}

Die glattmuskulären Tumoren unsicheren malignen Potenzials, welche entsprechend der englischsprachigen Abkürzung auch im Deutschen gerne als STUMP bezeichnet werden, stellen eine schlecht definierte Gruppe zwischen den benignen Leiomyomen und den malignen Leiomyosarkomen dar. Von einigen Expertengruppen wird der Begriff ,atypische glattmuskuläre Neoplasie" bevorzugt. Die STUMP-Kategorie sollte für jene wenigen Fälle vorbehalten sein, bei denen zwar besorgniserregende, auf Malignität verdächtige, für die Sarkomdiagnose aber nicht ausreichende histologische Merkmale vorliegen. Diese Kriterien sind laut Literatur bei eingeschränkter Evidenz mit einem erhöhten Rezidivrisiko vergesellschaftet (• Tab. 2). Leider blieb die Kategorie auch in der letzten WHO-Klassifikation schlecht definiert und beinhaltet streng genommen Fälle, die sich mit benignen Kategorien wie mitosereichen Leiomyome bzw. Leiomyome mit bizarren Zellkernen überschneiden bzw. diesen entsprechen (• Tab. 2). Ein STUMP liegt beispielsweise vor, wenn durch die Kombination unterschiedlicher Kriterien wie Atypiegrad und Mitoseindex, aber auch Tumorzellnekrose, keine eindeutige $\mathrm{Zu}$ ordnung in die Gruppe der Leiomyome bzw. der Leiomyosarkome möglich ist, aber auch wesentliche diagnostische Kriterien wie Atypien, Mitosegehalt oder die zelluläre Differenzierung nicht mit Sicherheit beurteilt werden können. Die STUMP-Kategorie zeigt die Komplexität der Diagnostik eines Teils der leiomyogenen Tumoren auf, auch die Tatsache, dass es dabei nicht nur Schwarz und Weiß, sondern zahlreiche Grauschat- 
Schwerpunkt: Gynäkopathologie

Tab. 2 Kriterien für atypische glattmuskuläre Neoplasien/STUMP (WHO 2014)

\begin{tabular}{|c|c|c|c|c|}
\hline $\begin{array}{l}\text { Tumorzell- } \\
\text { nekrosen }\end{array}$ & Zelluläre Atypie & $\begin{array}{l}\text { Mitosegehalt } \\
\text { per } 10 \mathrm{HPF}\end{array}$ & $\begin{array}{l}\text { Rezidivhäufigkeit } \\
\text { laut Literatur }\end{array}$ & Kommentar \\
\hline Fehlend & $\begin{array}{l}\text { Fokale/multifokale } \\
\text { mäßige bis starke } \\
\text { Atypie }\end{array}$ & $<10$ & $\begin{array}{l}14 \% \text { (3 von } 22 \text { Fäl- } \\
\text { len) }\end{array}$ & - \\
\hline Fehlend & $\begin{array}{l}\text { Diffuse mäßige bis } \\
\text { starke Atypie }\end{array}$ & $<10$ & $\begin{array}{l}10 \% \text { (7 von } 67 \text { Fäl- } \\
\text { len) }\end{array}$ & $\begin{array}{l}\text { DD zu Leio- } \\
\text { myomen mit } \\
\text { bizarren Zellker- } \\
\text { nen }\end{array}$ \\
\hline Vorhanden & Keine Atypien & $<10$ & $\begin{array}{l}26,7 \% \text { ( } 4 \text { von } \\
15 \text { Fällen) }\end{array}$ & - \\
\hline Fehlend & Keine Atypie & $\geq 15$ & Keine Rezidive & $\begin{array}{l}\text { DD zu mitose- } \\
\text { reichem Leio- } \\
\text { myom }\end{array}$ \\
\hline
\end{tabular}

Tab. 3 Sarkome des Endometriumstromas (ESS) und undifferenzierte Sarkome

\begin{tabular}{|c|c|c|c|}
\hline & Low-grade-ESS & High-grade-ESS & Undifferenziertes Sarkom \\
\hline Zytologie & Monomorph & $\begin{array}{l}2 \text { Komponenten: } \\
\text { rund-/spindelzellig }\end{array}$ & $\begin{array}{l}\text { Hochgradig atypisch, poly- } \\
\text { morph }\end{array}$ \\
\hline Wachstumsmuster & Infiltrativ & Infiltrativ & Expansiv \\
\hline Gefäßbezug & $\begin{array}{l}\text { Intravaskuläres } \\
\text { Wachstum }\end{array}$ & Gefäßinvasion & Gefäßinvasion \\
\hline Alter & 50-55 Jahre & 50-70 Jahre & 70-80 Jahre \\
\hline Prognose/Verlauf & Gut, späte Rezidive & $\begin{array}{l}\text { Schlecht, frühe Rezidi- } \\
\text { ve }\end{array}$ & $\begin{array}{l}\text { Schlecht, frühe Rezidive, } \\
\text { häufig Metastasen }\end{array}$ \\
\hline Östrogenbezug & $\begin{array}{l}\text { Östrogenabhängig } \\
\text { (ER-positiv) }\end{array}$ & $\begin{array}{l}\text { Partiell östrogen-ab- } \\
\text { hängig, ER-positiv } \\
\text { oder -negativ }\end{array}$ & $\begin{array}{l}\text { Östrogenunabhängig (ER- } \\
\text { negativ) }\end{array}$ \\
\hline Diagnosestellung & Hysterektomie & Kürettage/Biopsie & Kürettage/Biopsie \\
\hline $\begin{array}{l}\text { Molekular- } \\
\text { pathologie }\end{array}$ & JAZF1-SUZ12 & $\begin{array}{l}\text { YWHAE-NUTM2, } \\
\text { BCOR-Fusionen }\end{array}$ & TP53-Mutationen \\
\hline \multicolumn{4}{|c|}{ ER Östrogenrezeptor, ESS Sarkom des Endometriumstromas } \\
\hline
\end{tabular}

Tab. 4 Systematik der gemischten Tumoren des Uterus

\begin{tabular}{|c|c|c|}
\hline & Benigne epithelial & Maligne epithelial \\
\hline Benigne mesenchymal & $\begin{array}{l}\text { Adenofibrom } \\
\text { Adenomyom, APAM }\end{array}$ & $\left(\right.$ Karzinofibrom) $^{\mathrm{a}}$ \\
\hline Maligne mesenchymal & Adenosarkom & Karzinosarkom \\
\hline
\end{tabular}

tierungen gibt. Leider gibt es weder immunhistochemische noch molekulare Parameter, um zweifelhafte Fälle eindeutig als benigne oder maligne zu kategorisieren. STUMP zeigen ein niedriges Rezidivrisiko, wobei Rezidive spät auftreten.

\section{Tumoren des Endometrium- stromas und verwandte Tumoren}

In den letzten Jahren kam es zur Wiedereinführung der Kategorie des hochmalignen bzw. schlecht differenzierten („high-grade“) Sarkoms des Endometriumstromas (High-grade-ESS), dem die WHO 2014 auch Rechnung trägt [3]. Die High-grade-ESS wurden auf Basis der Histomorphologie und mo- lekularer Veränderungen neu definiert (- Tab.3). Sie sind durch eine rund- und spindelzellige Histomorphologie charakterisiert und können auch hinsichtlich des Wachstums- und Infiltrationsmusters durchaus den Low-grade-ESS ähneln (- Abb. 4 und 5). Besonders auffallend sind eine oft fibromyxoide Architektur und das Fehlen der dickwandigen, kleinen, arteriolenartigen Blutgefäße [17]. Tumoren mit myxoider Struktur müssen gegenüber myxoiden Leiomyosarkomen abgegrenzt werden, wobei letztere zum Teil Rearrangements im Bereich des PLAG1-Gens aufweisen, die aufgrund der erhöhten Immunreaktivität auch mittels Immunhistochemie nachgewiesen werden kann [18, 19]. Auf molekularer Ebene finden sich bei den ESS typischerweise Fusionsgene, die auch bei der Diagnostik hilfreich sein können. Für die High-grade-ESS finden sich neben der YWHAE-NUTM2(früher: FAM22)-Genfusion auch Fusionen und interne Tandemduplikate im Bereich des BCOR-Gens [20-23]. Die Fusionstranskripte können mittels Next Generation Sequencing (NGS) detektiert werden. Immunhistochemisch lässt sich neben BCOR auch Cyclin D1 nachweisen, häufig auch CD56 (NCAM) und CD99 [24, 25]. In der Praxis ist vor allem Cyclin D1 gut etabliert und verbreitet. Ein Teil der High-grade-ESS weist eine Positivität für Östrogenrezeptoren auf.

Die 2003 in der WHO etablierte und zuletzt umbenannte Gruppe der undifferenzierten Uterussarkome (früher: undifferenzierte Sarkome des Endometriums) scheint durch neuere Forschungsergebnisse zunehmend speziell in der Kategorie des High-grade-ESS aufzugehen. Sie wird in den nächsten Jahren unter Einbeziehung neuer molekulare Erkenntnisse neu definiert werden müssen.

Es gibt Überlegungen und Vorschläge, die Nomenklatur der Low-gradeStromaneoplasien zu modifizieren [26]. Low-grade-ESS und Stromaknoten weisen eine idente klein- und rundzellige Histomorphologie und ähnliche molekulare Alterationen auf, speziell im Bereich des JAZF1-Gens mit dem Auftreten von JAZF1-SUZ12(früher: JJAZ1)- Fusionen. Low-grade-ESS unterscheiden sich von Stromaknoten aber durch ihr myoinva- 

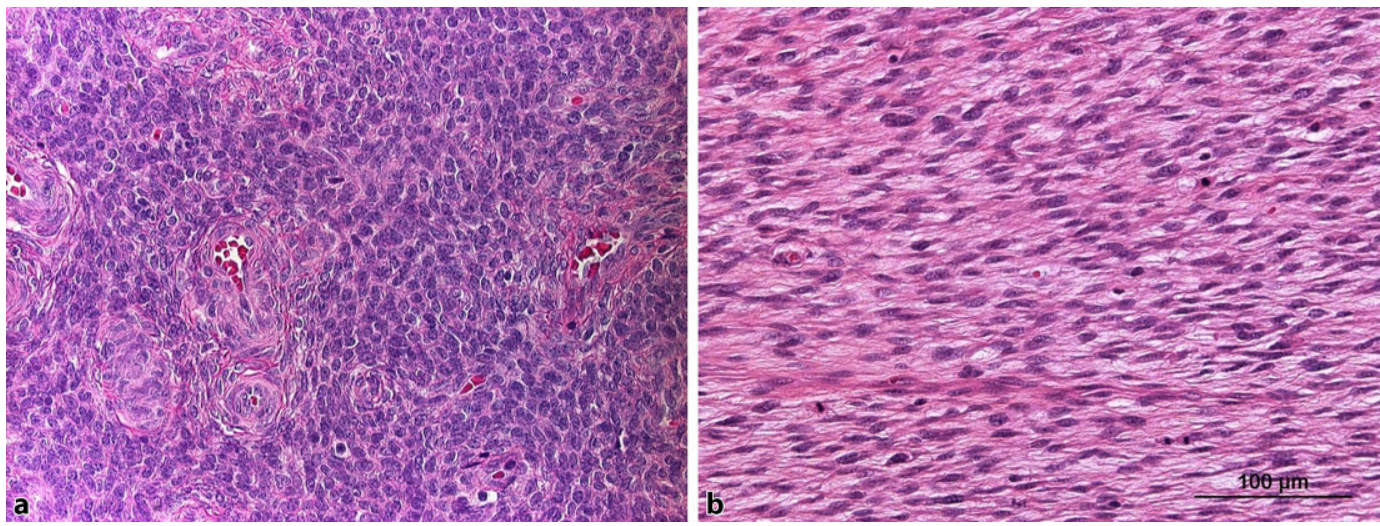

Abb. 4 A Unterschiedliche Histomorphologie von Low-grade-Neoplasien und High-grade-Sarkomen des Endometriumstromas (ESS): Für die Low-grade-Neoplasien (Stromaknoten und Low-grade-ESS) ist eine rundzellige Morphologie mit kleinen, arteriolenartigen Blutgefäßen typisch (a). High-grade-ESS können zum Teil eine spindelzellige Morphologie aufweisen, die arteriolenartigen Blutgefäße fehlen (b). HE-Färbung
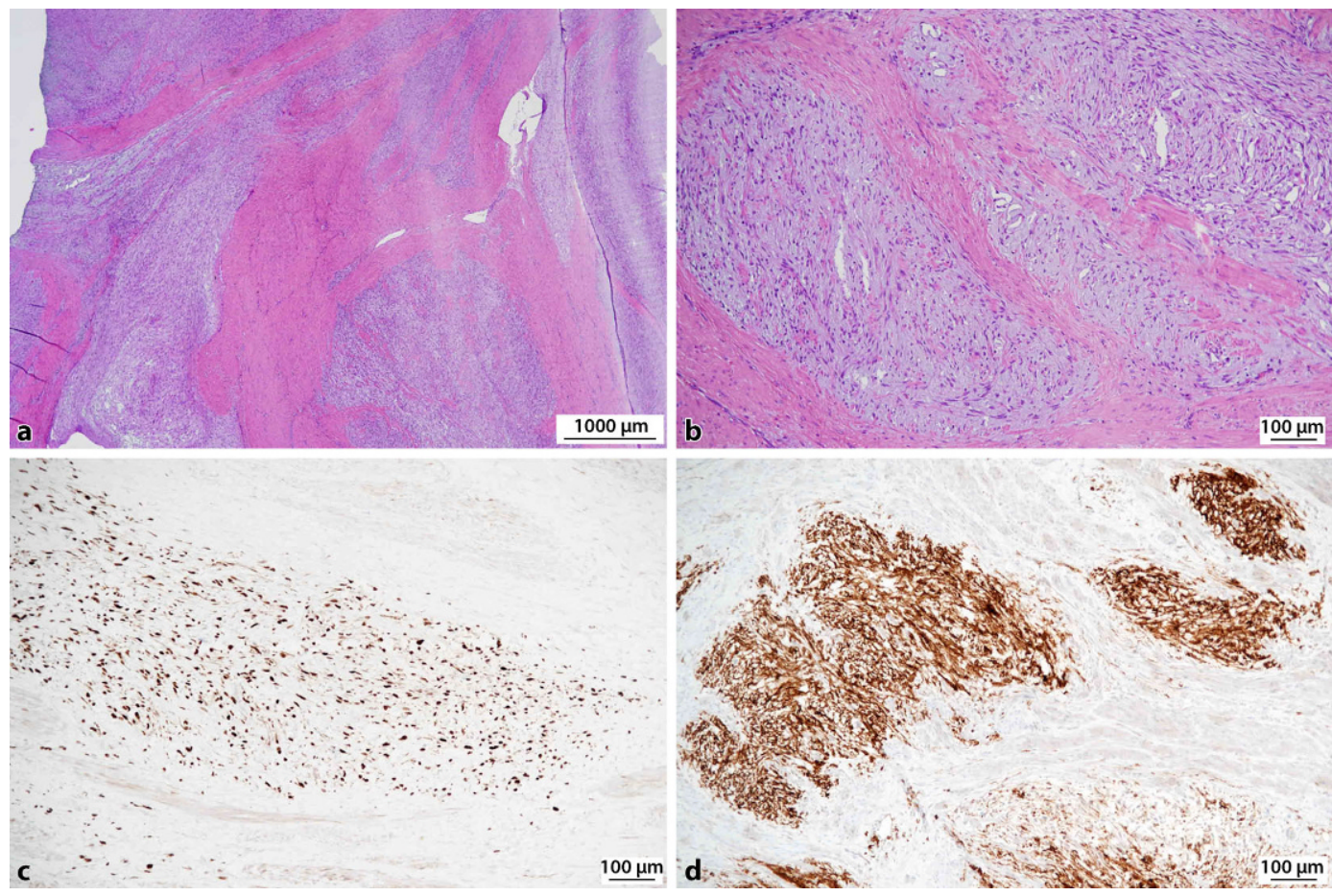

Abb. $5<$ Schlecht differenziertes Sarkom des Endometriumstromas (Highgrade-ESS) mit zungenförmiger Myometriuminvasion (a), fibromyxoidem Zellbild (b) und Immunreaktivität für Cyclin D1 (c) sowie CD10 (d). HE-Färbung (a, b) bzw. DAB-Färbung $(c, d)$

sives Wachstum. Die Abgrenzung des Stromaknoten vom Low-grade-ESS ist daher am Biopsie- und Kürettagematerial unmöglich, da eine Beurteilung des Verhaltens zum umgebenden Myometrium fehlt. Außerdem wurde für Lowgrade-ESS mit minimaler Invasion des Myometriums eine exzellente Prognose berichtet, allerdings ist die Evidenz für diese Tumoren gering.

Diagnostik und Differenzialdiagnostik der Low-grade-ESS und der Stromaknoten können in den meisten Fällen auf Basis von Histomorphologie und Immunhistochemie unter Einsatz eines Panels von Caldesmon und CD10, eventuell auch Desmin durchgeführt werden. Der Nachweis von Östrogen(ER) und Progesteronrezeptoren (PR) ist für Low-grade-ESS und Stromaknoten typisch und kann therapeutisch zu Nutze gemacht werden. Für die Diagnostik des High-grade-ESS ist der immunhistochemische Nachweis von Cyclin D1 hilfreich. High-grade-ESS können einen komplexen Immunphänotyp aufweisen mit unterschiedlicher Positivität für CD10, Cyclin D1 und ER/PR. Einen neuen therapeutischen Ansatz könnte eine gegen die Fusionsproteine zielgerichtete Therapie bzw. Immuntherapie darstellen [27].

\section{Gemischte leiomyogene und Stromatumoren}

Gemischte Tumoren des Endometriumstromas und der glatten Uterusmuskulatur (gemischte leiomyogene und Stromatumoren des Uterus) sind ebenfalls sehr selten, wobei jede Komponente zumindest $30 \%$ einnehmen sollte [28]. Die meisten dieser Tumoren sind benig- 

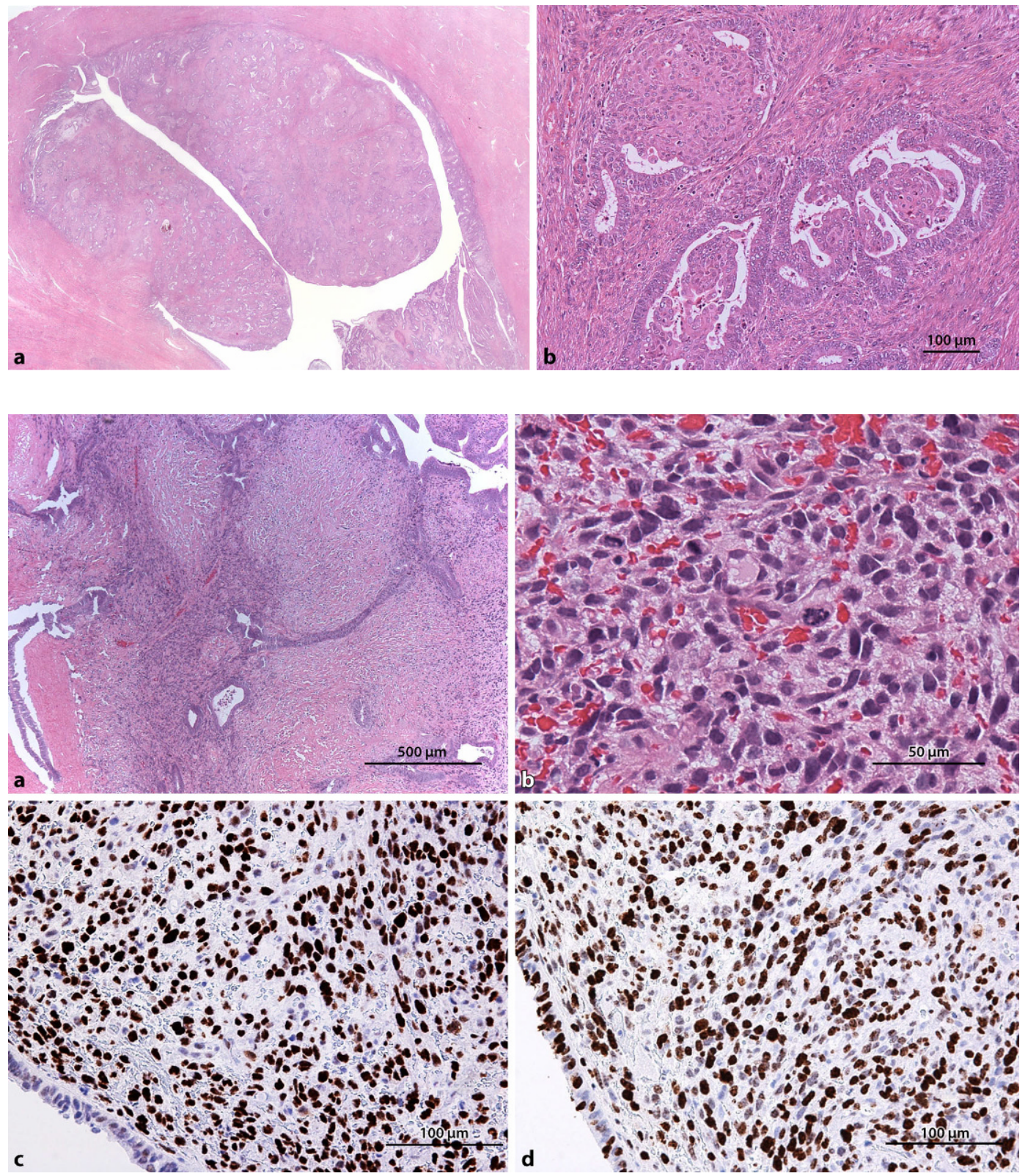

Abb. $6 \triangleleft$ Atypisches polypoides Adenomyom (APAM) als intrakavitärer Polyp (a), aufgebaut aus reichlich endometrioiden Drüsen mit Plattenepithelmetaplasie und einem myofibroblastären Stroma (b). HE-Färbung

\section{Abb. $7 \triangleleft$ Adenosar- kom mit hochmaligner sarkomatöser Kompo- nente (, sarcomatous overgrowth"), charakte- risiert durch erhöhten Zellgehalt (a,b), Atypi- en und Mitosen (b), eine mutationsspezifische Immunreaktivität für TP53 (c) und eine sehr hohen Ki67-Färbeindex. HE-Färbung $(a, b)$ bzw. DAB-Färbung $(c, d)$}

ne, basierend auf den zellulären Atypien und dem Verhalten zur Umgebung. In der WHO 2014 wurden diese Tumoren nicht mehr als eigene Entität geführt, sondern sollten entweder als Knoten des Endometriumstromas oder als Lowgrade-ESS mit glattmuskulärer Differenzierung klassifiziert werden [3].

\section{Keimstrangartiger Tumor des Uterus (UTROSCT)}

Der keimstrangstrangartige Tumor des Uterus wird derzeit als eine mit den Stromaneoplasien des Endometriums verwandte Entität eingestuft [3]. Die Abkürzung UTROSCT stammt von der englischen Bezeichnung „uterine tumor resembling ovarian sex cord tumor". Diese Tumorkategorie zeigt eine vielgestaltige Histomorphologie, die gegenüber Karzinomen, aber auch Low-grade-ESS, Stromaknoten und gemischten leiomyogenen Tumoren sowie Stromatumoren abgrenzt werden muss. Die Differenzialdiagnose zu Karzinomen kann speziell bei submuköser Lokalisation bedeutsam sein. Auch der Immunphänotyp ist heterogen mit wechselhafter Immunreaktivität für CD10, Desmin, Zytokeratin und CD99 [29]. Auf molekularer Ebene finden sich Genfusionen, die in den Stromatumoren des Endometri- ums fehlen (NCOA2/3) [30]. Hingegen fehlen im UTROSCT die für die Lowgrade-ESS und Stromaknoten typischen Genfusionen von JAZF1-SUZ12 [31]. Keimstrangartige Tumoren des Uterus zeigen in der Regel einen gutartigen Verlauf, ein ungünstiger Verlauf kann bei myoinvasivem Wachstum vorkommen [32].

\section{Gemischte epitheliale und mesenchymale Tumoren}

$\mathrm{Zu}$ den gemischten epithelialen und mesenchymalen Tumoren des Uterus zählen Adenomyome, Adenosarkome und Kar- 

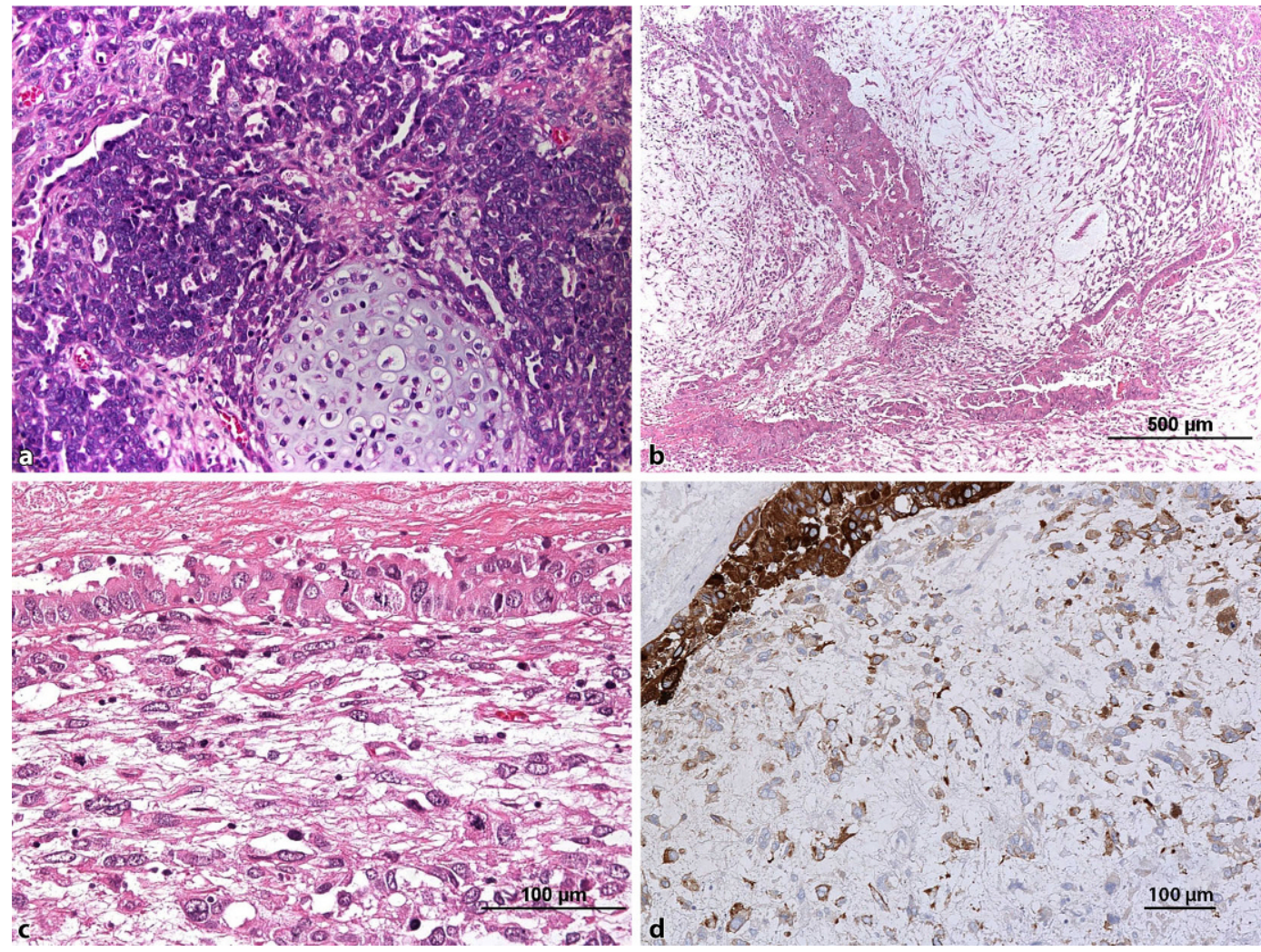

Abb. $8<$ Karzinosarkom mit seröser karzinomatöser und chondrosarkomatöser Komponente (a) bzw. endometrioider karzinomatöser und fibromyxosarkomartiger mesenchymaler Komponente (b). Epithelial-mesenchymaler Übergang (EMT) mit spindelzelliger Transformation in der undifferenzierten Komponente (c) und verändertem Reaktionsmuster für Zytokeratine in der Immunhistochemie (d). HE- bzw. DAB-Färbung zinosarkome (• Tab. 4; [33]). Die Entität der Adenofibrome ist nach wie vor stark umstritten, da die Abgrenzung zu Adenosarkomen mit geringen Atypien und geringer Zellularität kaum möglich ist. Diese Diagnose sollte daher mit Vorsicht gestellt werden. Adenosarkome und Karzinosarkome zeigen eine Assoziation mit Tamoxifentherapie, Karzinosarkome auch mit einer Langzeitöstrogenmedikation ohne Gestagenkomponente [34].

Adenomyome bestehen aus Adenomyoseherden innerhalb eines knotigen Myometriums, sind immer gutartig und diagnostisch unproblematisch. Die atypischen polypoiden Adenomyome (APAM) sind durch eine komplexe, aber organoide Architektur charakterisiert, die aus endometrioiden Drüsen und einem fibromuskulären bzw. myofibroblastären Stroma besteht (• Abb. 6). Die Drüsen stehen mitunter sehr dicht, weisen oft geringe Atypien auf und enthalten morulaartige Formationen metaplastischen Plattenepithels. Das Stroma ist immunhistochemisch positiv für SMA, aber negativ für Desmin und Caldesmon, wodurch der Name Adenomyofibrom besser passen würde
[35]. Der häufige Verlust von PTEN und häufige andere molekulare Veränderungen wie Mikrosatelliteninstabilität und KRAS-Mutationen legen die neoplastische Natur und die Verwandtschaft mit der atypischen Endometriumhyperplasie nahe [36]. Ein myoinvasives Wachstum fehlt. APAM sind gutartig, zeigen aber in etwa $10 \%$ der Fälle eine Assoziation mit einem meist gut differenzierten endometrioiden Adenokarzinom, wobei differenzialdiagnostisch vor allem ein konfluentes, labyrinthartiges Wachstumsmuster und eine klar erkennbare Myometriuminvasion zum Tragen kommen.

Für Adenosarkome sind die blattartigen Strukturen an der Oberfläche und die manschettenartige Verdichtung des Stromas um Drüsen diagnostisch wesentlich. Sie zeigen histologisch nur zu einem Teil eine Invasion des Myometriums [37]. Ihre quasi „niedrig maligne Potenz“ äußert sich durch Rezidive, insbesondere bei lokaler Abtragung und Uteruserhalt. Ein Übergang zum Low-grade-ESS mit glandulären Strukturen ist fließend und kann in speziellen Fällen schwierig sein. Ganz wesentlich ist bei den Adenosar- komen das Erkennen einer Überwucherung des Stromas durch ein High-gradeSarkom, was im Englischen sehr treffend als „sarcomatous overgrowth" bezeichnet wird (•Abb. 7). Dabei können sich unterschiedliche Formen der Differenzierung finden, meist eine fibrosarkomatöse, aber auch eine rhabdomyosarkomatöse Komponente. Häufig finden sich eine mutationsspezifische Immunreaktivität für TP53 und ein sehr hoher Ki67-Färbeindex. "Sarcomatous overgrowth" stellt auch den wesentlichen prognostischen Faktor dar.

Karzinosarkome werden biologisch als sarkomatoide oder sarkomatös differenzierte Karzinome betrachtet, aufgrund der histogenetischen Klassifikation aber von der WHO unter die gemischten Tumoren eingereiht. Sie sind auf molekularer Ebene klonal und können in Metastasen ein monomorphes, meist karzinomatöses Muster aufweisen. Der Begriff „Maligner Müller-Mischtumor" (MMMT) ist zwar als Synonym in der WHO-Klassifikation angeführt, dem Begriff „Karzinosarkom“ sollte aber der Vorzug gegeben werden [34]. Karzinosarkome zeigen typischerweise eine 

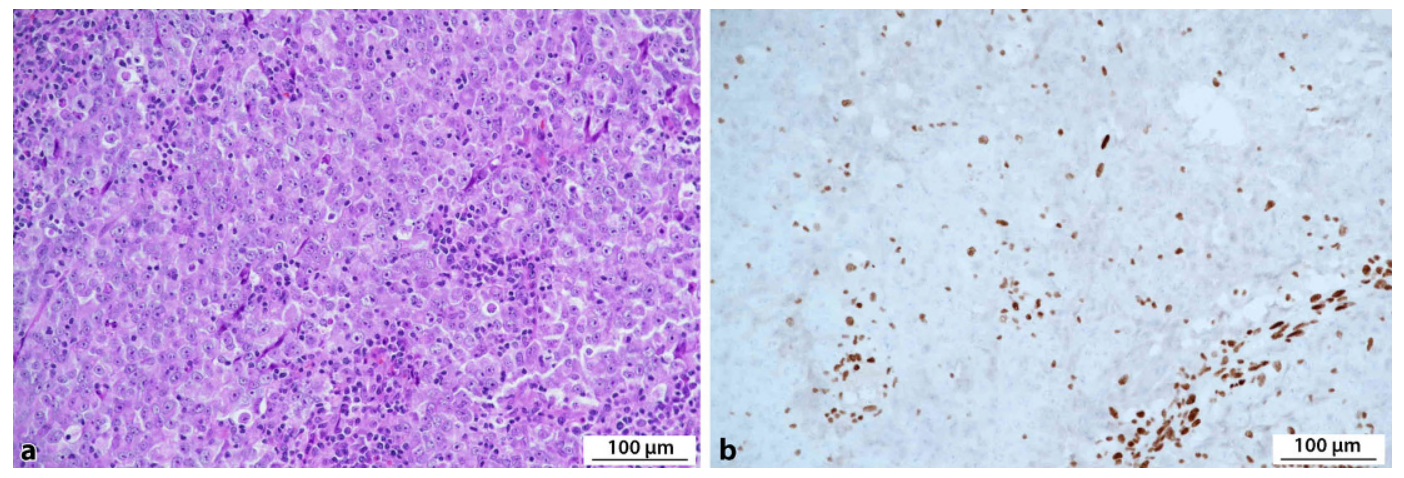

Abb. $9<$ Undifferenziertes Endometriumkarzinom mit rhabdoider Zellmorphologie (a, HE-Färbung) und fehlender Immunreaktivität für SMARCA4 in den Tumorzellen (b, DAB-Färbung)

intensive Mischung von meist schlecht differenzierten epithelialen und mesenchymalen Komponenten, wobei die epitheliale Komponente zum Teil auch gut differenziert sein kann (• Abb. 8). Die epitheliale Differenzierung kann oft ein gemischtes Bild aufweisen, mitunter ist sie nicht klar zuzuordnen. Die mesenchymale Komponente zeigt meist eine leiomyosarkomatöse oder fibrosarkomatöse Differenzierung, oft ist die Differenzierung ebenso wie die der epithelialen Komponente nicht sicher einzuordnen. Auch heterologe Elemente wie rhabdomyosarkomatöse, osteo- und chondrosarkomatöse Gewebe können vorkommen, seltener eine neuroektodermale Differenzierung. Ein wesentlicher Risikofaktor für das Auftreten von Metastasen ist die Eindringtiefe ins Myometrium [34]. Prognostisch ungünstig sind weiterhin eine nichtendometrioide karzinomatöse Komponente und heterologe Elemente speziell bei Tumoren im Stadium I. Differenzialdiagnostisch müssen die Karzinosarkome vom dedifferenzierten Subtyp des undifferenzierten Endometriumkarzinoms abgegrenzt werden, der aus einer völlig undifferenzierten und einer gut oder mäßig differenzierten karzinomatösen Komponente aufgebaut ist [38]. Dedifferenzierte Karzinome zeigen zu einem Teil einen Verlust von SMARCA4, speziell bei rhabdoider Zytomorphologie (• Abb. 9). Häufig sind diese SMARCA4defizienten, dedifferenzierten Endometriumkarzinome auch mikrosatelliteninstabil, bedingt durch einen Verlust von MLH1 in Folge Methylierung des Promotors. TP53 zeigt in der Regel eine Wildtyp-Färbereaktion, im Gegensatz zu Karzinosarkomen, bei denen TP53 meist mutiert ist. In einem kleinen Teil der dedifferenzierten Karzinome findet sich auch eine sarkomartige Differenzierung mit Verlust der Zytokeratinimmunreaktivität, wodurch die Abgrenzung zu Karzinosarkomen schwierig sein kann. Weiterhin können Karzinosarkome abschnittsweise ein ähnliches Gewebemuster aufweisen wie undifferenzierte bzw. dedifferenzierte Karzinome, sodass die Untersuchung zahlreicher Proben erforderlich sein kann.

\section{Fazit für die Praxis}

- Die Diagnose eines Leiomyosarkoms basiert auf zellulärer Atypie und hohem Mitosegehalt. Die Bedeutung der Tumorzellnekrose ist durch die WHO-Klassifikation 2014 in den Hintergrund getreten.

- Eine STUMP-Diagnose (STUMP, glattmuskuläre Tumoren unsicherer maligner Potenz) sollte selten gestellt und durch eine (externe) Zweitmeinung abgesichert werden. Es gibt Überschneidungen der STUMP-Definition mit bizarren und zellreichen Leiomyomen.

- Low-grade- und High-grade-ESS (ESS, Sarkom des Endometriumstromas) unterscheiden sich durch Histomorphologie, Immunphänotyp, charakteristische genomische Veränderungen und klinischen Verlauf.

- Bei Adenosarkomen sprechen eine stärkere Polymorphie und ein hoher Mitosegehalt für eine Überwucherung durch ein hochmalignes Sarkom.

- Karzinosarkome unterscheiden sich von undifferenzierten Endometriumkarzinomen durch ihre histolo- gische Heterogenität, die Differenzialdiagnose kann aber schwierig sein.

\section{Korrespondenzadresse}

Prim. Univ.-Prof. Dr. S. F. Lax

Institut für Klinische Pathologie und

Molekularpathologie, LKH Graz II, Standort

West, Akademisches Lehrkrankenhaus der Medizinischen Universität Graz Göstinger Straße 22, 8020 Graz, Österreich sigurd.lax@kages.at

Funding. Open access funding provided by Medical University of Graz.

\section{Einhaltung ethischer Richtlinien}

Interessenkonflikt. S. F. Lax gibt an, dass kein Interessenkonflikt besteht.

Dieser Beitrag beinhaltet keine vom Autor durchgeführten Studien an Menschen oder Tieren.

Open Access Dieser Artikel wird unter der Creative Commons Namensnennung 4.0 International Lizenz (http://creativecommons.org/licenses/by/4.0/deed. de) veröffentlicht, welche die Nutzung, Vervielfältigung, Bearbeitung, Verbreitung und Wiedergabe in jeglichem Medium und Format erlaubt, sofern Sie den/die ursprünglichen Autor(en) und die Quelle ordnungsgemäßnennen, einen Linkzur Creative Commons Lizenz beifügen und angeben, ob Änderungen vorgenommen wurden.

\section{Literatur}

1. Zaloudek C, Hendrickson MR, Soslow RA (2011) Mesenchymal tumors of the uterus. In: Kurman RJ, WEllenson LH, Ronnett BM (Hrsg) Blaustein's pathology of the female genital tract, 6 . Aufl. Springer, New York, S453-578

2. Wittekind C (Hrsg) (2018) TNM Klassifikation maligner Tumoren, 8. Aufl. Wiley-VCH, Weinheim

3. Oliva E, Carcangiu ML, Carinelli SG, Ip P, Loening T, Longacre TA, Nucci MR, Prat J, Zaloudek CJ (2014) Mesenchymal tumours of the uterine corpus. In: Kurman RJ, Carcangiu ML, Herrington S, Young RH 
(Hrsg) Tumours of the female reproductive organs. IARC, Lyon, S 135-147

4. Lax SF (2019) Mesenchymal tumors of the female genital tract. In: Raspollini MR, LopezBeltran A (Hrsg) Gynecologic and urologic pathology. Similarities, differences and challenges. Cambridge University Press, Cambridge

5. Bell SW, Kempson RL, Hendrickson MR (1994) Problematic uterine smooth muscle neoplasms. A clinicopathologic study of 213 cases. Am J Surg Pathol 18(6):535-558

6. Tavassoli FA, Devilee P (Hrsg) (2003) Tumours of the breast and female genital organs. WHO classification of tumours. IARC, Lyon

7. Atkins K, Bell SW, Kempson R, Hendrickson M (2001) Epitheloid smooth muscle tumors of the uterus. Mod Pathol 14(1):132A

8. Croce S, Ducoulombier A, Ribeiro A, Lesluyes T, Noel JC, Amant F, Guillou L, Stoeckle E, DevouassouxShisheboran M, Penel N, Floquet A, Arnould L, Guyon F, Mishellany F, Chakiba C, Cuppens T, Zikan $M$, Leroux A, Frouin E, Farre I, Genestie C, Valo I, MacGrogan G, Chibon F (2018) Genome profiling is an efficient tool to avoid theSTUMP classification of uterine smooth muscle lesions: a comprehensive array-genomichybridization analysis of 77 tumors. Mod Pathol 31(5):816-828. https://doi.org/10. 1038/modpathol.2017.185

9. Davidson B, Abeler VM, Hellesylt E, Holth A, Shih le $M$, Skeie-Jensen $T$, Chen L, Yang Y, Wang TL (2013) Gene expression signatures differentiate uterine endometrial stromal sarcoma from leiomyosarcoma. Gynecol Oncol 128(2):349-355. https://doi.org/10.1016/j.ygyno.2012.11.021

10. Lax S (2009) Mesenchymal uterine tumors. Leiomyomas. Pathologe 30(4):274-283. https:// doi.org/10.1007/s00292-009-1160-1

11. Croce S, Young RH, Oliva E (2014) Uterine leiomyomas with bizarre nuclei: a clinicopathologic study of 59 cases. Am J Surg Pathol 38(10):1330-1339. https://doi.org/10.1097/PAS.0000000000000249

12. Miettinen $M$, Felisiak-Golabek $A$, Wasag $B$, Chmara M, Wang Z, Butzow R, Lasota J (2016) Fumarase-deficient uterine leiomyomas: an immunohistochemical, molecular genetic, and clinicopathologic study of 86 cases. Am J Surg Pathol 40(12):1661-1669. https://doi.org/10. 1097/PAS.0000000000000703

13. Siegler $L$, Erber R, Burghaus S, Brodkorb T, Wachter D, Wilkinson $\mathrm{N}$, Bolton J, Stringfellow $\mathrm{H}$, Haller F, Beckmann MW, Hartmann A, Agaimy A (2018) Fumarate hydratase $(\mathrm{FH})$ deficiency in uterine leiomyomas: recognition by histological features versus blind immunoscreening. Virchows Arch 472(5):789-796. https://doi.org/10.1007/s00428018-2292-6

14. Colgan TJ, Pendergast S, LeBlanc M (1993) The histopathology of uterine leiomyomas following treatment with gonadotropin-releasing hormone analogues. Hum Pathol 24(10):1073-1077

15. Ip PP, Lam KW, Cheung CL et al (2007) Tranexamic acid-associated necrosis and intralesional thrombosis of uterine leiomyomas: A clinicopathologic study of 147 cases emphasizing the importance of drug-induced necrosis and early infarcts in leiomyomas. Am J Surg Pathol 31:1215-1224

16. Boyd C, McCluggage WG (2011) Unusual morphological features of uterine leiomyomas treated with progestogens.J Clin Pathol 64(6):485-489. https:// doi.org/10.1136/jcp.2011.089664

17. Ferreira J, Felix A, Lennerz JK, Oliva E (2018) Recent advances in the histological and molecular classification of endometrial stromal neoplasms.
Virchows Arch 473(6):665-678. https://doi.org/10. 1007/s00428-018-2470-6

18. Arias-Stella JA 3rd, Benayed R, Oliva E, Young RH, Hoang LN, Lee CH, Jungbluth AA, Frosina D, Soslow RA, Antonescu CR, Ladanyi M, Chiang S (2018) Novel PLAG1 gene rearrangement distinguishes a subset of uterine myxoid leiomyosarcoma from other uterine myxoid mesenchymal tumors. Am J Surg Pathol. https://doi.org/10.1097/PAS. 0000000000001196

19. Hoang LN, Aneja A, Conlon N, Delair DF, Middha S, Benayed R, Hensley ML, Park KJ, Hollmann TJ, Hameed MR, Antonescu CR, Soslow RA, Chiang $S$ (2017) Novel high-grade endometrial stromal sarcoma: a morphologic mimicker of myxoid leiomyosarcoma. Am J Surg Pathol 41(1):12-24. https://doi.org/10.1097/PAS.0000000000000721

20. Lee $\mathrm{CH}$, Marino-Enriquez $\mathrm{A}, \mathrm{Ou} \mathrm{W}$, Zhu $\mathrm{M}$, Ali RH, Chiang S, Amant F, Gilks CB, van de Rijn M, Oliva E, Debiec-Rychter M, Dal Cin P, Fletcher JA, Nucci MR (2012) The clinicopathologic features of YWHAE-FAM22 endometrial stromal sarcomas: a histologically high-grade and clinically aggressive tumor. Am J Surg Pathol 36(5):641-653. https://doi.org/10.1097/PAS.0b013e31824a7b1a

21. Chiang $\mathrm{S}$, Lee $\mathrm{CH}$, Stewart CJR, Oliva $\mathrm{E}$, Hoang LN, Ali RH, Hensley ML, Arias-Stella JA 3rd, Frosina D, Jungbluth AA, Benayed R, Ladanyi M, Hameed M, Wang L, Kao YC, Antonescu CR, Soslow RA (2017) BCOR is a robust diagnostic immunohistochemical marker of genetically diverse high-grade endometrial stromal sarcoma including tumors exhibiting variant morphology. Mod Pathol 30(9):1251-1261. https://doi.org/10. 1038/modpathol.2017.42

22. Lewis N, Soslow RA, Delair DF, Park KJ, Murali R, Hollmann TJ, Davidson B, Micci F, Panagopoulos I, Hoang LN, Arias-Stella JA 3rd, Oliva E, Young RH, Hensley ML, Leitao MM Jr., Hameed M, Benayed $R$, Ladanyi $M$, Frosina $D$, Jungbluth AA, Antonescu CR, Chiang S (2018) ZC3H7B$B C O R$ high-grade endometrial stromal sarcomas: a report of 17 cases of a newly defined entity. Mod Pathol 31(4):674-684. https://doi.org/10.1038/ modpathol.2017.162

23. Panagopoulos I, Thorsen J, Gorunova L, Haugom L, Bjerkehagen B, Davidson B, Heim S, Micci $F$ (2013) Fusion of the ZC3H7B and BCOR genes in endometrial stromal sarcomas carrying an X;22-translocation. Genes Chromosomes Cancer 52(7):610-618. https://doi.org/10.1002/ gcc. 22057

24. Lee $\mathrm{CH}$, Ali $\mathrm{RH}$, Rouzbahman $\mathrm{M}$, Marino-Enriquez A, Zhu M, Guo X, Brunner AL, Chiang S, Leung $S$, Nelnyk N, Huntsman DG, Blake Gilks C, Nielsen TO, Dal Cin P, van de Rijn M, Oliva E, Fletcher JA, Nucci MR (2012) Cyclin D1 as a diagnostic immunomarker for endometrial stromal sarcoma with YWHAE-FAM22 rearrangement. Am J Surg Pathol 36(10):1562-1570. https://doi.org/10. 1097/PAS.0b013e31825fa931

25. McCluggage WG, Lee CH (2018) YWHAENUTM2A/B translocated high-grade endometrial stromal sarcoma commonly expresses CD56 and CD99. Int J Gynecol Pathol. https://doi.org/10 1097/PGP.0000000000000554

26. Matias-Guiu X, Davidson B, Lax SF (2018) Can the classification of low-grade endometrial stromal tumors still be improved? Virchows Arch 473(6):663-664. https://doi.org/10.1007/s00428 018-2474-2

27. Tuyaerts S, Amant F (2018) Endometrial stromal sarcomas: a revision of their potential as targets for immunotherapy. Vaccines (Basel). https://doi.org/ 10.3390/vaccines6030056

28. Oliva E, Young RH, Amin MB, Clement PB (2002) An immunohistochemical analysis of endometrial stromal and smooth muscle tumors of the uterus: a study of 54 cases emphasizing the importance of using a panel because of overlap in immunoreactivity for individual antibodies. Am J Surg Pathol 26(4):403-412

29. Hurrell DP, McCluggage WG (2007) Uterine tumour resembling ovarian sex cord tumour is an immunohistochemically polyphenotypic neoplasm which exhibits coexpression of epithelial, myoid and sex cord markers. J Clin Pathol 60(10):1148-1154

30. Dickson BC, Childs TJ, Colgan TJ, Sung YS, Swanson D, Zhang L, Antonescu CR (2018) Uterine tumor resembling ovarian sex cord tumor: a distinct entity characterized by recurrent NCOA2/3 gene fusions. Am J Surg Pathol. https://doi.org/10.1097/ PAS.0000000000001153

31. Staats PN, Garcia JJ, Dias-Santagata DC et al (2009) Uterine tumors resembling ovarian sex cord tumors (UTROSCT) lack the JAZF1-JJAZ1 translocation frequently seen in endometrial stromal tumors. Am J Surg Pathol 33:1206-1212

32. Biermann K, Heukamp LC, Buttner R, Zhou H(2008) Uterine tumor resembling an ovarian sex cord tumor associated with metastasis. Int J Gynecol Pathol 27(1):58-60

33. Lax SF (2019) Mixed epithelial and mesenchymal tumors of the uterus. In: Raspollini MR, LopezBeltran A (Hrsg) Gynecologic and urologic pathology. Similarities, differences and challenges. Cambridge University Press, Cambridge

34. Wells M, Oliva E, Palacios J, Prat J (2014) Mixed epithelial and mesenchymal tumours of the uterine corpus. In: Kurman RJ, Carcangiu ML, Herrington S, Young RH (Hrsg) Tumours of the female reproductive organs. IARC, Lyon, S148-151

35. Soslow RA, Chung MH, Rouse RV, Hendrickson MR, Longacre TA (1996) Atypical polypoid adenomyofibroma (APA) versus well-differentiated endometrial carcinoma with prominent stromal matrix: an immunohistochemical study. Int J Gynecol Pathol 15(3):209-216

36. Nemejcova K, Kenny SL, Laco J, Skapa P, Stanek L, Zikan M, Kleiblova P, McCluggage WG, Dundr P (2015) Atypical polypoid adenomyoma of the uterus: an immunohistochemical and molecular study of 21 cases. Am J Surg Pathol 39(8):1148-1155. https://doi.org/10.1097/PAS.0000000000000428

37. Gallardo A, Prat J (2009) Mullerian adenosarcoma: a clinicopathologic and immunohistochemical study of 55 cases challenging the existence of adenofibroma. Am JSurg Pathol 33(2):278-288

38. Ramalingam P, Croce S, McCluggage WG (2017) Loss of expression of SMARCA4 (BRG1), SMARCA2 (BRM) and SMARCB1 (INI1) in undifferentiated carcinoma of the endometrium is not uncommon and is not always associated with rhabdoid morphology. Histopathology 70(3):359-366. https://doi.org/10.1111/his.13091 